\title{
Role of Arterial catheters as a source of Hospital related Bacteremia
}

\author{
Murtaza Mustafa, SC.Shimmi, MTH.Parash, MDS.Rahman, SS.Husain \\ Faculty of Medicine and Health Sciences, University Malaysia Sabah, Kota Kinabalu, \\ Sabah, Malaysia.
}

\begin{abstract}
Central line-associated bloodstream infections (CLABSIs) are common in intensive care units (ICUs) and in the medical patients. In the United States approximately 250.000 cases of bloodstream infections (BSIs) reported annually, associated with increase in longer hospital stay, costs and mortality. Primary bacteremia without local infection elsewhere, Including intravascular catheter sources account for approximately one half of ICU related bacteremias. The incidence of CLABSIs in non-ICU, general medical patients compare able to the rate in ICU patients. Sources of BSIs include contaminated fluids, catheter hub and lumen, and contaminated skin at catheter insertion site. Pathogens gain access into to the blood stream through extraluminal or intraluminal surface of the device. Bacterial bioflm is thought to be a virtually universal phenomena following insertion of intravascular device. Gram positive organisms, gram negative organisms and fungi is the frequently isolated BSIs pathogens. Multi drug resistant pathogens, and extended spectrum Blactamase (ESBLS) producing organisms, and bacteremia in elderly has high mortality rate. Molecular methods play important role in the diagnostic laboratory techniques. CLABSIs can be prevented by following $C D C$ 's guidelines for the prevention of device- related infections.
\end{abstract}

KEYWORDS: Device associated bacteremia, Blood stream infections, Diagnosis, Prevention.

\section{INTRODUCTION}

In 1977,Maki suggested that more than 25,000 patients develop device related bacteremia in the United States each year [1].Centers for Disease Control and Prevention(CDC) has estimated that approximately 80,000 central line-associated blood stream infection(CLABSIs) currently occur in intensive care units(ICUs) each year[2].Other researchers reported approximately 250,000 cases of blood stream infection(BSI) in the U.S. annually[3].Over the years the use of vascular access devices in medicine has resulted in even more complications associated with their use. The rates of bacteremia associated with the use of intravascular devices increased significantly into early 2000s. Recent data suggest that rates of CLABSIs may be decreasing, perhaps as a result of prevention programs implemented in many hospitals since 2001[4].An intense focus on prevention of health care associated infections, including CLABSIs, and requirements for the incorporation of performance measures into regulatory and financial reimbursement system has the potential to significantly reduce these infections[5,6].Device associated infections occur as sporadic cases as well as in case clusters caused by the same organism. Vascular catheters have become an increasingly important source of bacteremias, increasing from $3 \%$ in the mid-1970s to $19 \%$ in the early 1990s [7].Primary bacteremia(i.e., no apparent local infection elsewhere caused by the same organism),including intravascular catheter sources, now account for approximately one half of all ICU related bacteremias [8,9].In cancer patients,56\% of all bloodstream infections from 1999 to 2000 were CLABSIs[10].Menon et al., reported 21.4\% vascular catheters related nosocomial infections in one prospective study in the ICU patients [11].The problem of iatrogenic, device -associated bacteremia is not unique to the United States in one prospective study of bacteremia from Australia, nosocomial bacteremias accounted for $40 \%$ of all cases and half of the nosocomial cases were device associated[12].Marshall and colleagues reported that the incidence of CLABSIs in non-ICU, general medical patients was comparable to the rate in ICU patients[13].Edmond et al,. reported that gram-positive organisms accounted for $64 \%$ of cases, gram-negative organisms accounted for $27 \%$ and $8 \%$ were caused by fungi, in a surveillance for nosocomial blood stream infections at 49 hospitals over 3 years period[14].BSIs with multidrug resistant pathogens, and extended spectrum $\beta$ lactamase (ESBLs) producing pathogens, and bacteremia in elderly has high mortality rate[15,16].Inappropriate initial antimicrobial therapy is associated with adverse outcome in antibiotic-resistant gram-negative bacteremia, particularly patients with a high-risk source of bacteremia[17].CLABSIs can be prevented through proper management of central line and the techniques addressed in the CDC's Healthcare Infection Control Practices Advisory Committee(CDC/HIPAC) guidelines for the Prevention of Intravascular Catheter -Related Infections [18].The paper reviews blood stream infection associated with vascular catheters related infections. 


\section{SOURCES OF BLOOD STREAM INFECTION}

Contaminated fluids. Contamination of the fluid administered through the device is a major cause of epidemic intravenous device related bacteremias. None the less, infusate contamination is a rare cause of bacteremia. Infusion- related sepsis has been reviewed in detail, and both manufacture-related and in use contamination of infuse have been documented as causes of device-associated sepsis [19-21].Another factor influencing the pathogenesis of infuse-associated infection is the composition of the fluid. Different infusion fluids support the growth of differing pathogens. No infuscate is entirely free of risk; even sterile water for injection can support the growth of Burkholderia cepacia [22]. Parenteral nutrition solutions are superb substrates for the growth of certain microorganisms. Caseinhydrolysate solutions support the growth of many bacteria and fungi[23,24].Lipid emulsions support bacterial growth extremely well, and their use has also been associated with a risk for fungemia caused by the lipid- dependent yeast Malasseziafurfur, although not with contaminated infuscate[25,26],This risk has been primarily identified in the neonatal intensive care setting and has less been seen in the adults[26].The risk for coagulase-negative staphylococcus bacteremia in neonates has been directly linked to the administration of lipid infusions[27].Several additional outbreaks of bacteremias have been linked to compounding pharmacies that adhere to different quality control standards[28]. One national outbreak of Serritiamarcescens bacteremias occurred as a result of contaminated magnesium sulfate solution, and a second outbreak of Psedumonas putida and Stenotrophomonas maltophila was associated with contaminated heparin catheter-lock solution[28].

Parenteral nutrition solutions may also become contaminated during compounding in the hospital pharmacy [29].Two similar outbreaks of Candida parasilosis infections were linked to the backflow of yeasts into parenteral nutrition solution because the vacuum pumps were used improperly[29].The composition of the infuscate also influences the degree of irritation of the vascular intima at the site of infusion. Fluids that are not isotonic, those at nonphysilogic $\mathrm{pH}$, and those containing particulates all may irritate the vascular wall, thus provoking thrombus formation. Such thrombi may be seeded with microbes-either hematogenously or by direct extension [30].

Catheter hub and lumen contamination. Contamination of the catheter hub-infusion junction as a significant contributor to device-associated infection has been championed by Sitges-Serra and colleagues [31].These investigators suggested that endemic coagulase-negative staphylococcal bacteremias often rise as a result of contamination of the catheter hub with these organisms. A randomized study examining the effects of a redesigned protective hub found these hubs to be associated with significantly lower rate of catheter sepsis and culture positive catheter hubs, suggesting that the hub is a common portal of entry for bacteria[32]. Other investigators have incriminated the hub-tubing junction(particularly when it does not allow a good fit) in the pathogenesis of epidemics of coagulase-negative staphylococcal infection[33].Maki and Ringer found hub contamination to be the second most heavily weighed risk factor for catheter- associated infection in a large, prospective study[34] Salzman and colleagues noted that more than 50\% of episodes of central venous catheterrelated sepsis occurring in a neonatal ICU were preceded by colonization of the catheter hub with incriminating organism[35].In a subsequent experimental study, these investigators found that swabbing the catheter hub with disinfectant substantially reduced the hub's microbial burden and the preparation containing $70 \%$ ethanol were both more effective and more likely to be safer for the patient than preparations containing chlorhexidine [36]. Sherertz estimated that the hub, lumen or both contributed two third of the microorganisms that infected longterm catheters and one fourth of the microorganisms were from the skin[37].Finally, several outbreaks of bacteremia have been traced to contaminated medications-either those added directly to the system or those piggybacked into side port[38].Clusters of infection also have been listed to flushing catheters with fluids from a contaminated common source[39].

Conversely, some new technologies may be associated with increased risks for catheter-associated infection. Whereas the implementation of needless intravenous admixture system provide a safer work place environment for health care providers, some data suggest that use of these devices may be associated with increased risk for device associated infection [40].Multiple investigators of bacteremia outbreaks associated with needleless devices have suggested that the mechanism for bacteremia may involve contamination from end cap[41].Interestingly, different studies have paradoxically found either increased or decreased risk with the same needleless system(e.g., the interlink device[Baxter,Deerfield,11]), leading to conclusion that the primary risk associated with these devices is related to how the systems are used(e.g., frequency of changing end caps and adherence to recommended infection control procedures)rather than factors intrinsic to the system $[42,43]$.Appropriate staff education regarding use of these devices and ensuring compliance with manufacturer's recommendations is recommended to prevent device-related bacteremia[30]. 
Skin contamination at catheter insertion site. Many researchers favor the view that the catheter insertion tract provides the major avenue for the ingress of microbial invaders[1].Several studies have focused on microbial colonization around the catheter insertion site as a significant risk factor for catheter-associated infection[44].Supporting this contention are the studies of Cooper and Hopkins that demonstrated organisms on the exterior surface of catheters rather than within the catheter lumen[45].In the prospective study of Maki and Ringer, colonization around the catheter insertion site was the most strongly associated risk factor for local catheter infection[35]Similarly, Safdar determined that most catheter-related bacteremias occurring with shortterm noncuffed central catheters were extraluminally required and derived from cutaneous microflora [46]. Skin appears to be the primary source of intravenous device-related bacteremia for short-term catheters, placed for an average duration of less than 8 days[47].

Atela and co-workers conducted a prospective study to assess the turnover of superficial skin colonization by performing serial quantitative cultures of skin and catheter hub. Strains recovered from the targeted superficial skin sites demonstrated a poor correlation both with strains from previous skin cultures and with tip isolates [48]. Herwaldt and colleagues examined the source of coagulase-negative staphylococcal bacteremias in hematology-oncology patients and found that the same strain was identified in both skin and blood cultures in only 6 of 20 episodes. The strain was isolated only from other sites(primarily nares) in the remaining $70 \%$ of episodes, leading these investigators to the conclusion that mucous membranes might be a reservoir for strains of coagulase-negative staphylococcal causing bacteremia in immunocompromised patients .Importantly, these investigators were unable to identify colonization with the same strain for the majority of bacteremias; only 4 of 21 nosocomial bloodstream infections were preceded by colonization with the same strain. Most nosocomial coagulase -negative staphylococcal bacteremias in this study appeared to result from extrinsic introduction of the organism [49].

\section{PATHOGENESIS OF BACTEREMIA}

In intravascular device related bacteremia, microorganisms gain access into the blood stream through the extraluminal or intraluminal surface of the device. Microbial adherence and incorporation into biofilms then occur, resulting first in infection and then, in severe instances, in hematogenous dissemination [50]. There are various points of access to an intravascular device, each of which has been associated with both sporadic cases and case clusters of nosocomial bacteremia. Whereas the skin entry site has long been thought to be the most important portal of entry for invading microorganisms, the catheter hub-lumen has also been shown to be a major contributor to catheter-related bacteremia [51].The most common point of access appears to vary depending on the duration of time the catheter has been in place [30].

\section{PREVALENCE OF DEVICE - ASSOCIATED BACTEREMIA}

In 2006, rates of CLABSIs ranged from 1.5(inpatient medical/surgical wards) to $6.8 \%$ (in burn ICUs) bacteremias per 1000 central venous catheter days[4].Previous NNIS(National Nosocomial Infection Surveillance) rates from 1992 to June 2004 were higher. The previous medical ICU rate was 5.0 compared to $2.9 \%$ in the current report, perhaps indicating and actual reduction in the number of bacteremias[52].Intravenous device-related bacteremia rates are influenced by patient related parameters, catheter-related parameters,and hospital related parameters. Because of methodologic difficulties in performing appropriate scientific studies to characterize relative risk, many of these risk factors have been identified either retrospectively or in the epidemic setting. Still, each of the patient-related factors identified, has been associated with an increased risk of device-associated infection [53].

Alteration of patient's skin flora, either as a result of antimicrobial therapy or by colonization with an epidemic strain carried on the hands of hospital personnel, is a common event preceding catheter site infection. In addition, certain therapeutic devices (e.g. semipermeable membrane dressings) may actually increase the cutaneous microbial burden surrounding the catheter insertion site [54].Numerous epidemics of deviceassociated bacteremia have been linked to hospital personnel carrying an epidemic strain on their hands. Manipulating the system for repositioning, for obtaining a sample, or for any other reason increases the likelihood that catheter may become contaminated [55].Several catheter characteristics or properties have been suggested to be associated with an increased risk for catheter-associated infection. Catheters that irritate the vascular intima and provide thrombogenesis and the catheters that are made of materials that are intrinsically thrombogenic are likely to be associated with an increased risk for device associated infection [56].Older studies suggest that stiff catheters were associated with higher infection rates. Such catheters are thought to be more mobile in the insertion tract and thought to be more thrombogenic [57].A clear association has been established between the thrombogenecity of a catheter and the risk for device associated infection [58]. 
Despite differences in thromobogenecity, some authorities believe that all catheters become coated with fibrin sheath soon after placement. Currently, the majority of catheters are manufactured with antithrombogenic polymers, such as polyurethane[59].Catheter composition may influence the risk for infection in another way.Sheth and co-workers have shown that certain microorganisms ,most notably staphylococcus are able to adhere better to a catheter made from polyvinyl chloride than Teflon catheter[60].The physical size of the catheter(and therefore the size of the defect in the skin's intrinsic host defenses) is also likely to correlated with increased risk. Similarly, increasing the number of lumens in a catheter has been suggested to increase the risk for catheter-associated infection. Several studies have suggested that the use of multiple lumen catheters is associated with an increased risk for catheter -associated infection compared with the use of single lumen catheter [43].Not all studies have found this difference [61].The presence of distant infection resulting in the hematogenous seeding of the intravascular device has been incriminated [44].

Formation of a bacterial biofilm is now thought to be a virtually universal phenomena following insertion of intravascular devices [62].The microorganisms then embed themselves in and under the biofilm layer and become the source of intraluminal colonization and, eventually the sources of CLABSIs[63].Finally, the manner in which the catheter is used may influence risk. For example, risks for infection with pulmonary artery catheters may be higher because of the manner in which they are used [64]. Catheter management, including both insertion and maintenance, also may influence risk for infection. Several studies have shown that catheters placed by less experienced personnel are at increased risk for infection [65].Another study analyzed the efficacy of using a skilled team for placement of peripheral intravenous catheters [66].In this study, an intravenous therapy team significantly reduced both local and bacteremic complications associated with the placement of peripheral intravenous catheters, in part related to the timely placement of the catheters. Two studies suggest that insertion of central venous catheters with less than maximal sterile barriers increases the risk of catheter-related infection [46].

Several studies have suggested that the number of times the system is entered also influences the risk for infection [53].More than a single attempt to insert the catheter has also been found to be a risk factor for bacteremia. Insertion at a subclavian rather than a femoral site is clearly associated with a lesser risk of both infectious and thrombotic complications [67].In addition to patient-related risk factors, several hospitals related risk factors for CLABSIs have been either identified or proposed. In contraposition to patient-related factors, such as hospital related factors can often be altered for patient benefit. Nurse staffing variables, including nurse to-patient ratio, level of training, and permanent assignment to the unit("float" nurse vs regular unit staff nurse) have been shown to effect bacteremia rates[68].In addition, a number of studies have found that an education program focusing on risk factors and practice modification is associated with decreased rates of CLABSIs[69].

\section{MICROFLORA OF DEVICE-ASSOCIATED BACTEREMIA}

Frequently isolated pathogens in device-associated bacteremia include: coagulase negative staphylococci including staphylococcus epidermidis, Staphylococcus aureus, Enterococcus, Serritia marcescence, Candida spp, Candida albican, Pseudomonas aeruginosa, Enterobacter spp, Corynebacterium spp, (not C.diptheriae), and Acinetobacter baumannii.[30] Passereniet al in a study of nosocomial bloodstream infections, reported gram-positives are the main pathogens; there is no difference in etiology of catheter- related bloodstream infection (CRBSIs) between surgical and oncological patients. The lower incidence of grampostive non-CRBSIs in surgical patients was probably due to gram-negative infections secondary to surgical complications [69].Adolf reported that three most common causes of BSIs in the United States are coagulasenegative staphylococci, Staphylococcus aureus and enterococci. The emergence of vancomycin- resistant staphylococcal infections is of particular concern [70]..Klotz et al reported that. Candida species are the $4^{\text {th }}$ most common cause of nosocomial bloodstream infections in North America, of more than 8000 reported episodes of candidemia revealed high rates of polymicrobial infection occurring with candidemia [71].Staphylococci continue to predominate as the most frequently encountered pathogens in device related infections. Although S.aureus is a frequent cause of device-associated infection, coagulase-negative staphylococci have become the most common cause of these infections in the past two decades, especially in immunocompromised patients and those in whom long- term central venous access is required [72].Some institutions have observed a recent increase in catheter-associated infection caused by gram-negative bacilli[73].The past decade has witnessed an increasing occurrence of CLABSIs caused by multi resistant gram-negative bacilli, most notably Acinetobacterbaumannii.A. baumannii infections often occur in critically ill immunocompromised, highly antimicrobial agent-experienced patients who can ill effort any bacteremia, let alone one caused by multi-drug resistant bacteria [74,75].Patients with femoral catheters in one study had higher rates of gram-negative bacteremias and yeast related fungemias[76]. 


\section{VI.DIAGNOSTIC WORKOUT}

Clinical detection of catheter-associated septicemia is sometimes difficult. Clinical markers show a poor correlation with intravenous device-related bacteremia [77].Serum procalcitonin has been studied extensively as a marker of sepsis and one meta-analysis conducted that is differentiated bacterial from noninfected cases of systemic inflammation with greater accuracy than did C.reactive protein(CRP).Nonetheless, currently available assays often do not provide definitive results; many patients with bacteremia have indeterminate levels of procalcitonin [78].Using the semi quantitative culture technique, which defines a positive catheter tip culture as yielding 15 or more colonies, in combination of catheter-associated sepsis..Maki and colleagues reported a specificity in short peripheral catheters ranging between $76 \%$ to $96 \%$ and a positive predictive value of a positive catheter tip culture ranging between $16 \%$ and $31 \%$ in four studies [79].Farr and co-workers conducted a meta-analysis of catheter culturing techniques and suggested that the accuracy increases for catheter segment cultures with increasing quantitation (i.e.qualitative<semiquatitative <quantitative. The increase in accuracy is primarily due to increased specificity of the more quantitative tests[80].Although the relative merit of these various procedures remain to be definitively delineated, the ease of performing the semi quantitative technique described by Maki and co-workers has kept this procedure in widespread clinical use[79].Attempts to culture newer catheters with antimicrobial coating used for prevention of CLABSIs may lead to false-negative results[81]. Other investigators have suggested alternative techniques for diagnosing catheter-associated infections[80]. Acridine orange leukocyte cytospin testing of blood drawn through the catheter has been studies as a method of diagnosing infection while maintaining the catheter[82].During the last 15 years, molecular methods have begun to play an increasingly prominent role in the diagnostic microbiology laboratory.[30].Examples of usefulness of these techniques include the use of randomly amplified polymorphic DNA analysis for the rapid fingerprinting of coagulase-negative staphylococci, the use of other molecular typing methods(e.g., pulse-field electrophoresis and localization and/or probing the vicinity of the gene). and the molecular identification of antimicrobial resistance even before speciation can be completed. These molecular techniques are particularly valuable in epidemiologic investigations $[83,84]$.

\section{II.PREVENTION OF ARTERIAL-CATHETER RELATED BACTEREMIA}

CLABSIs can be prevented using simultaneously implementation of an array of practice improvements (i.e.,"bundles")[85].Education of health care personnel regarding standardized catheter insertion care, and prevention of infection has been shown to reduce the incidence of catheter -associated infections. In a metaanalysis, Safdar and Abad concurred that educational interventions can reduce rates of health care-associated infections[86].Catheter insertion checklists to ensure adherence to infection control practice have been used as part of the practice improvement bundle in a number of studies[85].Hand hygiene prior to catheter insertion or manipulation is an absolute requirement[86].CDC guidelines recommend disinfecting skin before catheter insertion and during dressing changes using tincture iodine and iodophors, $70 \%$ alcohol, or, preferably, a $2 \%$ chlorhexidine-based preparation[18].Even with the most stringent application of strategies to decrease catheterassociated infections, the presence of a vascular catheter remains a clear risk for infection. Thus, need for continued vascular access should be assessed at least daily, and nonessential catheters should be removed immediately [13]. Several investigators have advocated using prophylactic antimicrobials in specific defined circumstances to prevent catheter-associated infection. For example, Baier and colleagues found that prophylactic treatment of neonates with central line catheters with vancomycin effectively prevented coagulasenegative staphylococcal bacteremia associated with the use of these catheters[87]. The use of antimicrobial lock solutions, in which an antibiotic or other antimicrobial is injected into catheter lumen and the solution is left to dwell within lumen for a periods of some hours or days, has been examined as a method of preventing catheter infection during the past several years[88].New scientific approaches are needed to help establish optimal techniques for catheter management[52].

\section{CONCLUSION}

Vascular catheters-related bloodstream infections are common in the intensive care units and associated with high mortality rate. Reducing the device- associated infections is the most effective way to prevent CLABSIs.

\section{REFERENCES}

[1]. Maki D.Sepsis arising from Extrinsic Contamination of the infusion and measures for control.Lancaster,UK:MTP Press;1977.

[2]. 0'Grady NP,AlexanderM,DellingerEP, etal.Guidelines for the prevention of intravascular catheter-related infections.Infect Control Hosp Epidemiol.2002;23:759-69.

[3]. WisplinghoffH,BischoffT,TallentSM,etal.Noscomial bloodstream infections in US hospitals:analysis of 24,179 cases from prospective nationwide surveillance study. Clin Infect Dis.2004;39:309-17.

[4]. Edwards JR,PetersonKD,AndrusMI,etal.National Healthcare Safety Network (NHSN) Report,data summary for 2006,issued June 2007.Am J InfetControl.2007;35:290-301. 
[5]. Centers for Medicare and Medicaid Services. Hospital-Acquired conditions (Present on Admission Indicator) Available athttp://www.cms.hhs.gov/hosp/AcqCond/o6_Hospital-Hospital Acquired Conditionsasp\#TopOfPage.Accessed October 10. 2008.

[6]. The Joint Commission.2009 National Patient Safety Goals Hospital Program. Available at http://www .jointcommission .org/Patientsafety/ National PateientsafetyGoals/09_hapnpsgs.htm.AccessedOctober 10.2008.

[7]. Weinstein MP,TownsMI,QuarteySM,etal.The clinical significance of blood cultures in the 1990s: a prospective comprehensive evaluation of the microbiology,epidemiology and outcome of bacteremia and fungemia in adults.Clin InfectDis. 1997;24:584-602.

[8]. HugonnetS,Harbarth S, FerrieneK,etal.Bacteremic sepsis in intensive care: temporal trends in incidence organ dysfunction, and prognosis.Crit CareMed.2003;31:390-94.

[9]. RenaudB,Brun-Buisson C.ICU-Bacteremia StudyGroup.Outcomes of primary and catheter-related bacteremia:achohort and case control study in critically ill patients.Am J RespirCrit Care Med.2001;163:1584-90.

[10]. RaadJ,Hachem R, Hanna H,et al. Sources and outcomes of bloodstream infections in cancer patients: the role of central venous catheters.Eur J ClinMIcrobiol InfectDis.2007;26:649-56.

[11]. Menon Jayaram,MurtazaMustafa,G.Sannasey.A study on nosocomial infection in ventilated patients in the intensive care unit of Queen Elizabeth hospital ,Kota Kinabalu,Sabah,Malaysia.SciInt(Lahore).2002;4(2):143-50.

[12]. McGregor AR,CollignonPJ.Bacteremia and fungemia in an Australian general hospital-associations and outcomes. Med $J$ Aust.1993;158:671-74.

[13]. Marshal J,MermelIA,ClassenD,etal.Strategies to prevent central line-associated bloodstream infections in acute care hospitals. Infect Control HospEpidemiolol. 2008;29:S22-S30.

[14]. Edmond MB,WallaceSE,McClishDK,etal.Nosocomial bloodstream infections in United States hospitals: a three years analysis.Clin Infect Dis.1999;29(2):239-44.

[15]. ShaabanHA,EnasAD,MohammedSB,etal.Nosocomial bloodstream infection in intensive care units at Assiut university hospital(upper Egypt) with special reference to extended spectrum $\beta$ - lactamase producing organisms.BMCResearcNotes.2009;2:76-83.doi:10-1186/1756-0500-2-76

[16]. RebeloM,PereiraB,LimaJ,etal.Predictors of in-hospital mortality in elderly patients with bacteremia admitted to an internal medicine ward.Int Arch Med.2011;4(1):33.

[17]. Kang CI,KimSH,ParkWB,etal.Bloodstream infections caused by antibiotic-resistant gram-negative bacilli:risk factors for mortality and impact of inappropriate initial antimicrobial therapy on outcome.Antimicrob Agents Chemother.2005;49 \{2):760-6.

[18]. O'Grady NP,AlexanderM,BurnsLA,etal.Guidelines for the prevention of intravascular catheter-related infections,2011.Clin Infect Dis.2011;52(a):1087-99.

[19]. Maki D.Nosocomial bacteremia an epidemiologic overview.Am J Med.1981;70:719-732.

[20]. Maki D,RhmeF,MackelD,etal.Nationwide epidemic of septicemia caused by contaminated intravenous products:1 Epidemiologic and clinical features. Am JMed.1976;60:471-85.

[21]. StammW,CollelaJ,AndersonM,etal.Indwelling arterial catheters as a source of nosocomial bacteremia: an outbreak caused by Flavobacteriumspp.NEngl JMed.1975;292:1099-1102.

[22]. Douce RW, ZuritaJ,Sancher 0,et al.Investigation of an outbreak of central venous catheter-associated bloodstream infection due to contaminated water. Infect ControlHosp Epidemiol.2008;29:364-66

[23]. Goldman D, MartinW, Worhing tonJ. Growth of bacteria and fungi in total parenteral nutrition solutions. Am J Surg.1973;126:31418.

[24]. Maki D.Growth properties of microorganisms in infusion fluid and method of detection.In:PhillipsJ,ed.Microbiologic Hazards of Intravenous Therapy .Lancaster, UK:MTP Press;1977;13-47.

[25]. Jarvis W,HighsmithA,Bacterial growth and endotoxin production in lipid emulsion.JClin Microbiol.1984;19:17-20.

[26]. Dankoner W, Spector S,FiererJ.Malasseziafungemia in neonates and adults:complication of hyperalimentation.Rev Infect Dis.1987;9:743-837.

[27]. Avila-Figueroa C,GoldmanDA,RichardsonDK,etal.Intravenous lipid emulsions are the major determinant of coagulase-negative staphylococcal in very low birth weight newborns.Pediatr Infect Dis J.1998;17:10-17.

[28]. Souza Dias MB,HabertAB,BorrascaV,etal.Salvage of long term central venous catheters during an outbreak of Pdeudomonasputida and Stenotrophomonasmaltophila infections associated with contaminated heparin catheter-lock solution.Infect Control Hosp Epidemiol.2008;29:125-130.

[29]. PlloufieJ,BrownD,SivaJ,etal.Nosocomial outbreak of Candida paralosisfungemia related to intravenous infusion.ArchInt Med.1977;137:1686-89.

[30]. BeekmannSE,HendersonDK.Infections caused by percutaneous intravenous devices.In:Mandell,Douglas and Bennett's Principles and Practice of InfectiousDiseases, $7^{\text {th }}$ ed,MandellGI,BennettJE,Dolin R(editors)Churchill Livingstone Elsevier,2010.

[31]. Sitges-Serra A,PuigP,JurrietaF,etal.Hub contamination as the initial step in an outbreak of catheter-related spsis due to coagulase negative staphylococci during parenteral nutrition.JParenter enteral Nutr.1984;8:668-72.

[32]. Segura M,Alvarez Lerma F,TelladoJM,etal.A clinical trial on the prevention of catheter-related sepsis using a new hub model.Ann Surg.1996;223:363-69.

[33]. Pemberton I,LymanB,MandalJ,etal.Outbreak of Staphylococcus epidermidis nosocomial infections in patients receiving total parenteral nutrition Parenter Enteral Nutr.1984;8:325-326.

[34]. Maki D,RingerM.Evaluation of dressing regimen for prevention of infection with peripheral intravenous catheters gauze, a transparent polyurethane dressing and adiodophore transparent dressing.JAMA.1987;258:2396-2403.

[35]. SalzmanMB,IsenbergHD,ShapiroJF,etal.A prospective study of the catheter hub as the portal of entry for the organisms causing catheter-related sepsis in neonates.J Infect Dis.1993;167:487-490.

[36]. SalzmanM,IsenbergH,RubinI.Use of disinfectants to reduce microbial contamination of the hubs of vascular catheters Clin Microbiol.1993;31:475-479.

[37]. SheretzRJ.Pathogenesis of vascular catheter-related infections.In:SeifertH,JansenR,FarrBM,eds.Catheter-related Infections.NewYork:Marcel Dekker;1997;1-29.

[38]. GrohskopIA,RothVR,FeikinDR,etal.Serritialiquefaciens bloodstream infection from contamination of epoetinalfa at a hemodialysis center.NEngl J Med.2001;344:1491-97.

[39]. Van LaerF,Raes D, VandammeP,etal.An outbreak of Burkholderiacepecia with septicemia on a cardiology ward. Infect Control Hosp Epidemiol.1998;19:112-113.

[40]. Rupp ME,SholtzIA,JourdanDR,etal.Outbreak of bloodstream infection temporally associated with the use of an intravascular needless valve.Clin InfectDis. 2007; 44:1408-1414.

[41]. Cookson ST,Ihrig M,0'Mara EM,et al.Increased bloodstream infection rates in surgical patients associated with variation from recommended use and care following implementation of a needleless device. Infect Control Hosp Epidemiolol.1998;19:23-27. 
[42]. Danzig IE,ShortIJ,,CollinsK,etal.Bloodstrem infection associated with a needleless intravenous system in patients receiving home infusion therapy. JAMA. 1995: 273: 1862-64.

[43]. Do An, Ray BJ,BanerjeeSN,etal.Bloodstream infection associated with needleless device use and importance of infection control practices in the home health care setting.J Infect Dis.1999;179:442-48.

[44]. Biornson H, Colley R, Bower R,etal.Association between microorganisms growth at the catheter insertion site and colonization of the catheter in patients receiving total parenteral nutrition.Surgery.1982;92:720-27.

[45]. Cooper G,Hopkin C. Rapid diagnosis of intravascular catheter associated infection by direct Gram-staining of catheter segments'Engl J Med.1985;18:1142-50.

[46]. SafdarN,Maki DG. The pathogenesis of catheter-related bloodstream infection with nocuffed short- term central venous catheters. Intensive Care Med.2004;30:62-67.

[47]. Flowers RH,SchwenzerKJ,KopelRF,etal.Efficacy of an attachable subcutaneous cuff for the prevention of intravascular catheterrelated infection.JAMA.1989;261:878-83.

[48]. AtelaI,CollR,RelloJ,et al. Serial surveillance cultures of skin and catheter hub specimens from critically ill patients with central venous catheters: molecular epidemiology of infection and implications for clinical management and research.JClin Microbiol.1997;35:1784-90.

[49]. Herwaldt IA, Hollis RI, BoykenID,etal.Molecular epidemiology of coagulase-negative staphylococci isolated from immunocompromised patients. Infect ContHospEpidemiol.1992;13:86-92.

[50]. MarrieT, CostetonJW. Scanning and transmission electron microscopy of in situ bacterial colonization of intravenous and intraarterial cathetersClinMicrobiol. 1984;19:687-93.

[51]. CheesbroughJS,Finch RG, Burden RP.A prospective study of the mechanisms of infection associated hemodialysis catheters. $J$ Infect Dis.1986;154:579-89.

[52]. Centers for Disease Control and Prevention.National Nosocomial infections Surveillance (NNIS) system report, data summary from January 1992 to June 2004,issued October 2004.Am Infect Control.2004;32:440-85.

[53]. SafdarN,KlugarDM,Maki DG.A review of risk factors for catheter- related bloodstream infection caused by percutaneously inserted non cuffed central venous catheters: implications for preventive strategies.Medicine.2002;81:466-79

[54]. Kelsey M,Gosling M.A comparison of the morbidity associated with occlusive and non-occlusive dressing applied to peripheral intravenous device.JHosp Infect. 1984;5:313-21.

[55]. Lucas JW,BergerAM,FitzgeraldA, et al. Nosocomial infections in patients wity central catheters.JIntraven Nurs.1992;15:44-48.

[56]. RaadI,LanaM,KhalilSA,etal.The relationship between the thrombotic and infectious complications of central venous cathetersJAMA.1994;271:1014-16.

[57]. Welch G,Mckeel D, Jr,SilversteinP, etal.The role of catheter composition in the development of thrombophelibitis.SurgGynecol Obstet.1974;138:421-24

[58]. StillmanR,SolimanS,GarciaI, et al. Etiology of catheter associated sepsis.Arch Surg.1977;112:1497-99.

[59]. BozzettiF.Central venous catheter sepsis.SurgGynecol Obstet.1985;161:293-301.

[60]. ShethN,FransonT,RoseH,etal.Colonization of bacteria on polyvinyl chloride and Teflon intravenous catheters in hospitalized patients.JClin Microbiol.1983;18:1061-63.

[61]. Tan IH, Hess B,DiazIK,etal.Survey of the use of peripherally inserted central venous catheters in neonates with critical congenital cardiac disease.Cardiol Young. 2007; 17:196-201

[62]. RaadII,FangX,KentgenXM, etal.the role of chelators in preventing biofilm formation and catheter-related bloodstream infections.CurrOpin Infect Dis.2008;21:385-92.

[63]. DonlanRM,CostertonJW.Biofilms: survival mechanisms of clinically relevant microorganisms. ClinMicrobiol Rev.2002;15:167-93

[64]. HamtonA,SheretzR.Vascular- access infections in hospitalized patients.SurgClinNorth Am.1988;68:57-71.

[65]. Armstrong C,Mayhall C, Miller K,etal.Prospective study of catheter replacement and other risk factors for infection of hyperalimentationcatheters.J Infect Dis.1986; 154: 808-16.

[66]. SoiferNE,BorzakS,Edlin BR, et al.Prevention of peripheral venous catheter complications with an intravenous therapy team:a randomized controlled trial. Arch Intern Med.1998;158:473-77.

[67]. Hamilton HC,FoxcroftDR,Central venous access sites for the prevention of venous thrombosis, stenosis and infection in patients requiring long term intravenous therapy. Cochrane Database Syst Rev.2007;CD00484

[68]. FridkinSK,PearSM,WilliamsonTH,et al. The role of understanding in central venous catheter-associated bloodstream infections.Infect Control HospEpidemiol. 1996; 17: 150-8.

[69]. Rosenthal VD,GuzmanS,PezettoSM,etal.Effect of an infection control program using education and performance feedback on rates of intravascular device-associated bloodstream infections in intensive care units in Argentina. Am J InfectControl.2003;51:405-9.

[70]. Adolf WK.Nosocomial bloodstream infections:organisms,riskfactors,and implications.Clin Infect Dis.2000;31(supp14):S139S143.doi:101086/314079

[71]. Klotz SA,ChasinBS,PowellB,etal.Polymicrobial bloodstream infections involving Candida species: analysis of patients and review of the literature.DiagMicrobiol InfectDis.2007;59(4):401-6

[72]. Schulin T, Voss A.Coagulase-negative staphylococci as a cause of infections related to intravascular prosthetic devices: limitations of present therapy.ClinMicrobiolInfect.2001;7:1-7.

[73]. CastagnolaE,ConteM,VenzanoP,etal.Broviac catheter related bacteremias due to unusual pathogens in children with cancer: case reports and literature review.J Infect.1997;34:215-18.

[74]. MarchiamD,Zaidenstein R, LazarovitchT,etal.Epidemiology of bacteremia episodes in a single center: increase in Gram-negative isolates, antibiotic resistance and patients age.Eur J ClinMicrobiol Infect Dis.2008;27:1045-51.

[75]. Shih MJ, Lee Ny,LeeHC,etal.Risk factors of multidrug resistance in nosocomial bacteremia due to Acinetbacterbaumannii: a casecontrol study.JMicrobiolImmunolInfect.2008;41:118-23.

[76]. LorenteI,JimnezA,SantanaM,etal.Microorganisms responsible for intravascular catheter-related bloodstream infection according to catheter site. Crt CareMed. 2007;35:2424-27.

[77]. MermelIA,FarrRM,SherertzRJ,et al Guidelines for the management of intravascular cstheter-related infections.JInterven Nutr.2001;24:180-205.

[78]. Simon I,Gauvin F, AmreDK,etal.Serumprocalcitonin and C-reactive protein levels as markers of bacterial infections:a systematic review of meta-analysis.Clin InfectDis.2004;39:206-17.

[79]. Maki D,Weise C, Sarafin H.A semiquantitative method for identifying intravenous catheter- related infection.NEngl J Med.1977;296:1305-09.

[80]. SiegmanIgraY,AnglimAM.ShapiroDE,etal.Diagnosis of vascular catheter-related bloodstream infection: a meta -analysis.JClin Microbiol.1997;35:928-36. 
[81]. SchierholzJm,BachA,FleckC,etal.Measurement of ultrasonic-induced chlorohexidine liberation of activity of chlorhexidinesulfadiazine impregnated catheters in agar roll technique and broth culture Hosp Infect.2000;44:141-45.

[82]. Bong JJ,KiteP,AmmoreiBJ,etal.The use of a rapid in situ test in the detection of central venous catheter-related bloodstream infection:a prospective study.JParenterEnternal Nutr.2003;27:146-50.

[83]. BingenF,BarcMC,BrahimN,etal.Randomly amplified polymorphic DNA analysis provides rapid differentiation of methicillin resistant coagulase-negativestaphylococcus bacteremia isolates in pediatric hospital.JClinMicrobiol. 1995; 33:1657-59.

[84]. DominguzMA,LinaresJ,PulidsA,etal.Molecular tracking of coagulase-negative staphylococcal isolates from catheter-related infections.Microb DrugResist. 1996; 2:423-29.

[85]. BerenholtzSM,PronovostPJ,LipsetPA,etal.Eliminating catheter-related bloodstream infections in the intensive care unit..Crit Care Med.2004;32:2014-20.

[86]. SafdarN,Abad C. Educational interventions for prevention of healthcare-associated infection: a systematic review.Crit Care Med.2008;36:933-40.

[87]. Ruschulte H, Franke M,GastmeierP,etal.Prevention of central venous catheter-related infections with chlorhexidine gluconate impregnated wound dressing: a randomized controlled trial. Am Hematol.2008;88:267-72.

[88]. CarratalaJ, NiuboJ,FernandezSevillaA,etal.Randomized double-blind trial of an antibiotic-lock technique for prevention of grampositive central venous catheter-related infectioninneutropenic patients with cancer.Antimicrobial AgentsChemother.1999;43:220004. 